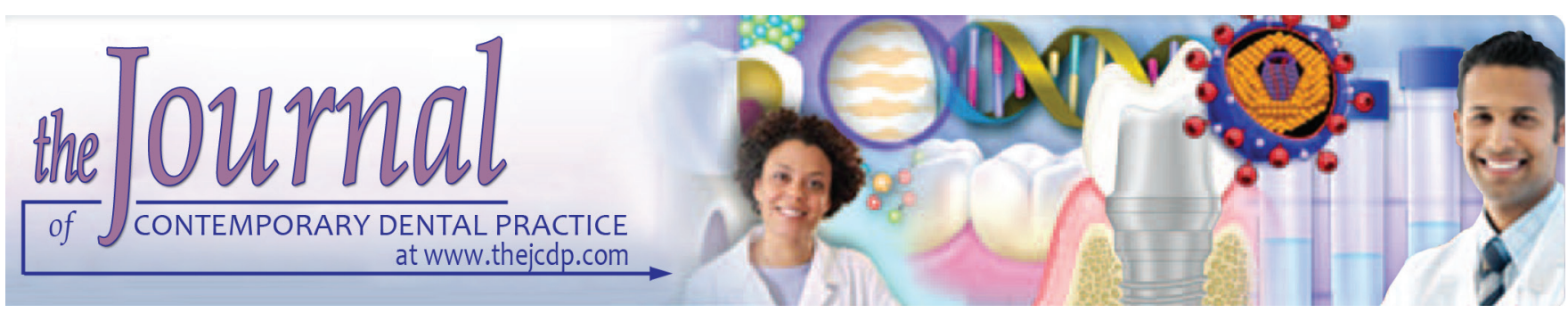

\title{
Herpes Simplex 1 and Periopathogen Role in Peri-implantitis
}

\author{
${ }^{1} \mathrm{~S}$ Parthiban, ${ }^{2}$ Nizar Ahmed, ${ }^{3} \mathrm{~T}$ Ramakrishnan, ${ }^{4} \mathrm{~V}$ Balakumar, ${ }^{5}$ Manoj Raja, ${ }^{6} \mathrm{Himanshu} \mathrm{Shekhar}$
}

\begin{abstract}
Introduction: The objectives of this study were to compare the qualitative and quantitative profiles of herpes simplex virus type I (HSV-1) in implant surfaces between participants with periimplantitis (PI) and Healthy peri-implant tissues and to quantitatively assess the relation between HSV-1 and periopathogens inside the microbiological profile associated with PI.
\end{abstract}

Materials and methods: A total of 40 patients with $\mathrm{PI}$ and 40 with healthy peri-implant tissues $(\mathrm{HI})$ were recruited. Plaque samples from peri-implant sulcus and internal implant connections were analyzed using quantitative real-time polymerase chain reaction to detect and quantify HSV-1 and periodontopathogens. Frequencies of detection and levels of microorganisms were compared between $\mathrm{PI}$ and $\mathrm{HI}$; the frequencies and levels of periodontopathogens were compared between HSV-1+ and $\mathrm{HSV}-1-\mathrm{PI}$ to assess qualitative relations between HSV-1 and bacteria. Correlation between HSV-1 and periodontopathogens levels was assessed in $\mathrm{PI}$ and $\mathrm{HI}$.

Results: A total of 77 dental implants affected by PI, and $113 \mathrm{HIs}$ were included. The HSV-1 prevalence was slightly higher in PI compared with controls (33.3 vs $23.8 \%$; $p>0.05$ ); HSV-1 was detected in external samples more frequently compared with internal samples. The HSV-1-positive patients revealed higher median loads of Prevotella intermedia $(\mathrm{Pi})$ and Campylobacter rectus $(\mathrm{Cr})$ compared with $\mathrm{HSV}$-1-negative patients. In the PI group, a significant positive correlation was evidenced between HSV-1 and Tannerella forsythia, Parvimonas micra $(\mathrm{Pm})$, Fusobacterium nucleatum, and $\mathrm{Cr}$ levels, while in the $\mathrm{HI}$, positive correlation between HSV-1 and Aggregatibacter actinomycetemcomitans, $\mathrm{Pi}$, and $\mathrm{Pm}$ was established.

Conclusion: The HSV-1 prevalence cannot be used to identify PI. The HSV-1 was found in similar levels of $\mathrm{PI}$ and $\mathrm{HI}$

\footnotetext{
1,2,5,6 Department of Periodontics, Thai Moogambigai Dental College, Chennai, Tamil Nadu, India

${ }^{3}$ Department of Periodontics, Adhiparasakthi Dental College Melmaruvathur, Tamil Nadu, India

${ }^{4}$ Department of Prosthodontics, Sathyabama University Dental College and Hospital, Chennai, Tamil Nadu, India

Corresponding Author: Himanshu Shekhar, Department of Periodontics, Thai Moogambigai Dental College, Chennai, Tamil Nadu, India, e-mail: shekhar.tdc@gmail.com
}

patients after an average of 6 years of loaded implants. The HSV-1 prevalence cannot be used to identify implants with or without the presence of $\mathrm{PI}$.

Clinical significance: Although HSV-1 is detected in PI site, HSV-1 may represent an unspecific indicator for the host response to the bacterial challenge observed in $\mathrm{PI}$.

Keywords: Herpes simplex virus type I, Periodontopathogens, Peri-implantitis, Real-time polymerase chain reaction.

How to cite this article: Parthiban S, Ahmed N, Ramakrishnan T, Balakumar V, Raja M, Shekhar H. Herpes Simplex 1 and Periopathogen Role in Peri-implantitis. J Contemp Dent Pract 2017;18(5):399-404.

\section{Source of support: Nil}

\section{Conflict of interest: None}

\section{INTRODUCTION}

Peri-implantitis (PI) is the most commonly encountered complication of dental implants. It is characterized by an inflammatory lesion of the peri-implant tissues induced by bacteria and is associated with loss of supporting bone. ${ }^{1}$ The microbiological picture with PI is predominately Gram-negative and anaerobic and, hence, resembles the microbiota associated with periodontitis., ${ }^{2,3}$ However, some differences in the composition of the microbiota between the two disease entities have also been seen. ${ }^{4}$ However, despite these high similarities between the microbiota of periodontitis and PI, peri-implant lesions are characterized by bony lesions that may extend in close proximity to the alveolar bone compared with the extent of the lesions in periodontitis, where a connective tissue capsule usually lines the lesion. ${ }^{5}$

The prevalence of herpes simplex virus type I (HSV-1) and Epstein-Barr virus (EBV) is reported to be associated with periodontitis and PI. ${ }^{6,7}$ However, the pathogenetical mechanisms are still not clearly understood. ${ }^{8}$ The prevalence of herpes viruses is extremely high and 
accounts for $95 \%$ of adults at the age of 30 years and older. ${ }^{9}$ Hence, viruses might provide favorable conditions for periodontopathogens and herpes viruses might enhance microbial and immunological dysbiosis in PI and periodontitis. ${ }^{10}$

Recent studies analyzed the frequency of EBV infection among different conditions of peri-implant tissues with controversial results; some of them reported positive associations between EBV and PI, ${ }^{11}$ while others did not. ${ }^{12}$ None has been done with HSV-1 association with PI.

The recently proposed keystone pathogen hypothesis of periodontal disease emphasized that qualitative characteristics in composition of the microbiota between disease and health are not substantially different, but that changes in proportions of pathogens represent the crucial distinguishing determinants. ${ }^{13}$ It is hypothesized that in PI, herpes viruses could create a microbiological profile in synergism with periodontopathogens characterized by their mutual increase. Hence, the aim of this study was to evaluate the role of HSV-1 prevalence at external and internal implant surfaces in patients with PI or healthy peri-implant tissues.

\section{MATERIALS AND METHODS}

This study was designed as a case-control study qualitatively and quantitatively estimating the profile of HSV-1 and periodontopathogens at internal and external implant surfaces of patients suffering from PI and participants with healthy peri-implant tissues.

The study was performed in Thai Moogambigai Dental College in the Department of Periodontology in the outpatient department with patients' informed consent and ethical clearance from Dr M.G.R. Educational and Research Institute.

About 40 patients with PI and 40 participants with healthy peri-implant tissues were enrolled in the study.

Patients were clustered into a case $(n=40)$ and a control group ( $n=40)$ according to the following criteria:

- PI cases were defined as those implants presenting with clinical probing depth (PD) $\geq 5 \mathrm{~mm}$, positive bleeding on probing (BOP), and recorded radiographic bone loss involving $\geq 2$ threads compared with the radiograph taken at the time of prosthetic placement.

- Healthy implant controls (HIs) were defined when $\mathrm{BOP}=0, \mathrm{PD} \leq 3 \mathrm{~mm}$, and no radiological bone loss were observed.

The participants of the study were recruited if being systemically healthy, nonsmokers, and lacking any of the exclusion criteria: (1) Previous periodontal treatment in preceding year; (2) intake of antibiotics in the preceding 6 months; (3) pregnancy or lactation in females.

\section{Clinical Outcome Variables and Radiographic Examination}

A full mouth periodontal and peri-implant examination was performed in all patients recording the following clinical parameters at six points per implant using a periodontal probe graded in mm (UNC15, Hu-Friedy Mfg. B.V, Rotterdam, the Netherlands):

- Clinical PD in mm

- Bleeding on probing measured 15 seconds after probing and recorded as present (1) or absent $(0)^{14}$

- Visible plaque accumulation (PI) measured along the mucosal margin and recorded as present (1) or absent $(0) .{ }^{15}$

Radiographs were taken from all the implant sites using a paralleling technique combined with the long cone.

\section{Microbiological Sampling}

The subgingival plaque samples were collected for microbiological analyses using commercial sampling kits (Geno Sen ${ }^{\circledR}$ RT-PCR diagnostic test, India). Briefly, before subgingival plaque sampling, each implant was isolated with cotton rolls. Absorbent paper points were inserted into the perio-implant pockets. After 15 seconds, these paper points were removed and placed into a $2 \mathrm{~mL}$ tube. After the submucosal plaque analyses, to collect the samples of the implant connection, prostheses and abutments were carefully removed, avoiding contamination as far as possible. Paper points were inserted for 30 seconds in the connection surface of the abutment. The tubes containing the sample were sent to a specialized laboratory (Veterinary College Diagnostic Lab, Kattupakkam) in the provided mailing envelopes.

Quantitative real-time polymerase chain reaction (RT-PCR) was performed to detect the presence/ absence and quantify the HSV-1 and bacterial pathogens: Aggregatibacter actinomycetemcomitans (Aa), Porphyromonas gingivalis (Pg), Tannerella forsythia (Tf), Treponema denticola (Td), Prevotella intermedia (Pi), Parvimonas micra (Pm), Fusobacterium nucleatum (Fn), Campylobacter rectus (Cr), Eikenella corrodens (Ec), and Candida albicans (Ca).

First, the total HSV-1 deoxyribonucleic acid (DNA) was isolated using reagents according to the manufacturer's guidelines (QIAxtractor ${ }^{\circledR}$ DNA Plasticware and QIAxtractor ${ }^{\circledR}$ DX Reagents [Qiagen, India ]). Then, RT-PCR was carried out for HSV-1 using the HSV-1 virus quantitative RT-PCR kit (Biotech - Bangalore) and a thermal cycling system (Rotor-Gene ${ }^{\circledR} \mathrm{Q}$ thermal cycling system [Qiagen, India]). Briefly, quantitative RT-PCR assays were performed in a volume of $25 \mu \mathrm{L}$ composed of $12.5 \mu \mathrm{L}$ of DNA mastermix (MasterMix Optima Multiplex 2X DNA [Diagenode, India]), $2.5 \mu \mathrm{L}$ 
of primers (Diagenode HSV-1 primers and double-dye probe [Diagenode, Liege, Belgium]) FAM (the most commonly used fluorescent dye attachment for oligonucleotides and is compatible with most fluorescence detection equipments), emission $520 \mathrm{~nm}$ ), $2.5 \mu \mathrm{L}$ of internal control DNA (Diagenode, Liege, Belgium], $2.5 \mu \mathrm{L}$ of internal control primers and double-dye probe (yellow dye, emission $548 \mathrm{~nm}$ ) (Diagenode, Liege, Belgium), and $5 \mu \mathrm{L}$ of DNA extract or HSV-1-positive control or HSV1-negative control or DNA standard (for quantitative standard curve) (MasterMix Optima Multiplex 2X DNA [Diagenode, India]). Five HSV-1 DNA dilutions were used for the standard curve (from 200 copies to 2,000,000 copies of HSV-1 amplicon/PCR reaction).

For bacteria analyses, quantitative RT-PCR assays were performed in a volume of $10 \mu \mathrm{L}$ composed of activator $\left(1 \times\right.$ QuantiFast ${ }^{\circledR}$ SYBR ${ }^{\circledR}$ Green PCR (Qiagen, Liepzig, Germany), $2 \mu \mathrm{L}$ of DNA extract, and $1 \mu \mathrm{M}$ of each primer. Species-specific $\mathrm{PCR}$ primers were used Metabion $\mathrm{GmbH}$ (Martinsried, Germany). Program for bacteria: $95^{\circ} \mathrm{C}$ for 30 seconds, followed by 40 cycles of 10 seconds at $95^{\circ} \mathrm{C}$, 10 seconds at $60^{\circ} \mathrm{C}$, and 35 seconds at $72^{\circ} \mathrm{C}$. A final melt curve analysis $\left(70-95^{\circ} \mathrm{C}\right.$ in $1^{\circ} \mathrm{C}$ steps for 5 seconds increments) was done. The program for HSV- 1 used was: $50^{\circ} \mathrm{C}$ for 2 minutes, $95^{\circ} \mathrm{C}$ for 10 minutes, followed by 45 cycles of 15 seconds at $95^{\circ} \mathrm{C}$, and 60 seconds at $60^{\circ} \mathrm{C}$.

FAM produce fluorescence signals, emission $520 \mathrm{~nm}$ and yellow dye, emissions were measured every cycle at the end of the extension step.

\section{Statistical Analysis}

For all the patients enrolled, descriptive analysis of each characteristic was $48 \mathrm{~nm}$, indicated as median with range (minimum-maximum) or frequencies (percentages).

Demographic variables including age, distribution of initial periodontal diagnosis, and gender were compared using Fisher's exact test. Full-mouth measurements including PI, BOP, and PD were calculated by patient and then by group. Analyses were conducted at the patient level; the comparison among internal/external implant surfaces was conducted at the implant level. When more than one implant for the same patient was present, a mean value was calculated and used in the analysis.

Clinical and microbiological variables did not present a normal distribution when tested with Shapiro-Wilk Levene's test of homogeneity of variance; therefore, intergroup comparison was performed using the MannWhitney test, while the $\mathrm{p}$ values were adjusted using Bonferroni post hoc test. Furthermore, the frequencies of HSV-1 and periodontopathogens between the groups as well as between the internal and external implant portions were assessed using a chi-square $\left(\chi^{2}\right)$ test. The correlations between HSV-1 levels and periodontopathogens in the groups as well as the correlation between HSV-1 and clinical parameters were assessed with Spearman's rank correlation test.

The statistical analysis was performed using commercial software (Statistical Package for the Social Sciences; SPSS 20.0, Inc., Chicago, Illinois, USA) with the significance level set at $\alpha=0.05$.

\section{RESULTS}

In the total sample of 80 participants ( 39 females, 41 males, mean age $63.3 \pm 9.3$ years), no statistically significant differences were found between genders and age. Twenty-one females and 19 males were reported in the group with PI. Nineteen females and 21 males made up then the healthy patient group HI.

A total of 77 dental implants affected by PI and 113 implants with healthy peri-implant conditions were included in the study (mean time of loading $6.25 \pm 1.6$ years).

Table 1 reports differences in bacterial loads between $\mathrm{HI}$ and PI. The levels of $\mathrm{Td}, \mathrm{Pi}, \mathrm{Fn}$, and $\mathrm{Cr}$ were significantly increased in PI when compared with HI patients, while the detection frequency of HSV-1 was only slightly increased in PI patients. However, the difference was not statistically significant.

Table 1: Comparison of viral and bacterial loads between $\mathrm{PI}$ and healthy controls

\begin{tabular}{llll}
\hline & Healthy $(n=42)$ & $P I(n=42)$ & $p$-value \\
\hline Herpes virus-1 (\%) & $10(23.8 \%)$ & $14(33.3 \%)$ & 0.47 \\
$\mathrm{Aa}$ & $0.00(0.00-194625.00)$ & $0.00(0.00-1162.50)$ & 0.99 \\
$\mathrm{Pg}$ & $134054.13(0.00-31771687.50)$ & $85500.00(0.00-104017325.00)$ & 0.52 \\
$\mathrm{Tf}$ & $200631.25(0.00-8797668.75)$ & $690450.00(0.00-39758926.25)$ & 0.18 \\
$\mathrm{Td}$ & $33281.25(0.00-7585500.00)$ & $270187.50(0.00-70125000.00)$ & $010^{*}$ \\
$\mathrm{Pi}$ & $367186.50(0.00-197115000.00)$ & $1935515.63(0.00-432071250.00)$ & $0.022^{*}$ \\
$\mathrm{Pm}$ & $448853.25(0.00-22893750.00)$ & $701812.50(444.50-114000000.00)$ & 0.09 \\
$\mathrm{Fn}$ & $1057187.50(3420.00-32460000.00)$ & $3976328.50(25273.25-141000000.00)$ & $027^{*}$ \\
$\mathrm{Cr}$ & $288922.50(0.00-13758000.00)$ & $768500.00(0.00-0265000.00)$ & $0.048^{*}$ \\
$\mathrm{Ec}$ & $65470.31(0.00-59355000.00)$ & $192000.00(0.00-188250000.00)$ & 0.15 \\
$\mathrm{Ca}$ & $0.00(0.00-3448500.00)$ & $0.00(0.00-143801250.00)$ & 0.41 \\
\hline${ }^{*} \mathrm{p}<0.05$ & & &
\end{tabular}


Table 2: Differences in evaluated pathogens between internal and external implants (results are expressed as count with percentage and median with range)

\begin{tabular}{lllc}
\hline & External & Internal & $p$-value \\
\hline Group (\%) & $113(61.7)$ & $73(52.9)$ & 0.14 \\
Healthy & $70(38.3)$ & $65(47.1)$ & \\
PI & & & $<0.001^{*}$ \\
HSV-1 (\%) & $145(79.2)$ & $132(95.7)$ & \\
Negative & $38(20.8)$ & $6(4.3)$ & $5.8(2.4-14.1)$ \\
Positive & & & $<0.001^{*}$ \\
OR (95\% Cl) & $0.00(0.00-3240000.00)$ & $0.00(0.00-9450.00)$ & 0.85 \\
HSV-1 & $0.00(0.00-389250.00)$ & $0.00(0.00-2325.00)$ & $<0.001^{*}$ \\
Aa & $42225.00(0.00-621750000.00)$ & $0.00(0.00-75750000.00)$ & $<0.001^{*}$ \\
Pg & $90000.00(0.00-228000000.00)$ & $978.75(0.00-18450000.00)$ & $0.001^{*}$ \\
Tf & $0.00(0.00-221250000.00)$ & $0.00(0.00-120750000.00)$ & $<0.001^{*}$ \\
Td & $492750.00(0.00-862500000.00)$ & $0.00(0.00-433500000.00)$ & $0.001^{*}$ \\
Pi & $276750.00(0.00-186750000.00)$ & $68775.00(0.00-52425000.00)$ & $<0.001^{*}$ \\
Pm & $1417500.00(0.00-264750000.00)$ & $138750.00(0.00-50025000.00)$ & $<0.001^{*}$ \\
Fn & $300750.00(0.00-106500000.00)$ & $0.00(0.00-15900000.00)$ & $<0.001^{*}$ \\
Cr & $55725.00(0.00-188250000.00)$ & $0.00(0.00-43650000.00)$ & 0.15 \\
Ec & $0.00(0.00-286500000.00)$ & $0.00(0.00-192000000.00)$ & \\
Ca & & & \\
\hline
\end{tabular}

$\mathrm{Cl}$ : Confidence interval; OR: Odds ratio

The levels of HSV-1 and periodontopathogens among internal and external implant portions in the groups are depicted in Table 2. The HSV-1 showed significantly increased frequencies as well as increased viral and bacterial loads at external implant when compared with internal implant portions with the exception of Aa and $\mathrm{Ca}$.

The HSV-positive patients revealed higher median loads $(\mathrm{p}<0.05)$ of Pi and $\mathrm{Cr}$ compared with HSV-negative patients (Table 3).
Table 4 depicts correlations between HSV-1 and periodontopathogen levels in PI and HI patients. In the PI group, significantly positive correlations were found between HSV and Tf, Pm, Fn, and Cr levels, while in the HI, a positive correlation between was established between EBV and Aa, Pi and Pm.

The analysis of the correlations between HSV-1 levels and clinical parameters did not demonstrate any significant differences.

Table 3: Levels of periopathogens between HSV-1-positive and -negative subjects

\begin{tabular}{llll}
\hline & Herpes virus-1- & Herpes virus-1+ & $p$-value \\
\hline Group (\%) & & & \\
Healthy & $32(53.3)$ & $10(41.7)$ & 0.47 \\
PI & $28(46.7)$ & $14(58.3)$ & 0.49 \\
Aa & $0.00(0.00-1162.50)$ & $0.00(0.00-194625.00)$ & 0.62 \\
Pg & $85500.00(0.00-104017325.00)$ & $116606.25(0.00-20790000.00)$ & 0.09 \\
Tf & $165000.00(0.00-18782500.00)$ & $752812.50(0.00-39758926.25)$ & 0.09 \\
Td & $46297.50(0.00-70125000.00)$ & $219187.50(0.00-18300000.00)$ & $0.041^{*}$ \\
Pi & $391700.00(0.00-432071250.00)$ & $4780221.88(0.00-375750000.00)$ & 0.22 \\
Pm & $448853.25(0.00-25578750.00)$ & $1187132.00(444.50-114000000.00)$ & 0.13 \\
Fn & $1163167.80(3420.00-42798750.00)$ & $3180087.50(25273.25-141000000.00)$ & $0.033^{*}$ \\
Cr & $224512.50(0.00-23384375.00)$ & $1012031.25(0.00-40265000.00)$ & 0.09 \\
Ec & $71079.38(0.00-28725000.00)$ & $369000.00(0.00-188250000.00)$ & 0.83 \\
Ca & $0.00(0.00-47875000.00)$ & $0.00(0.00-143801250.00)$ & \\
\hline
\end{tabular}

*Statistically significant

Table 4: Correlation coefficients between levels of HSV-1 and evaluated periopathogens in PI and healthy peri-implant tissues

\begin{tabular}{lllllllll}
\hline & Groups & $A a$ & $P g$ & $T f$ & $T d$ & $P i$ & $P m$ & $F n$ \\
\hline \multirow{3}{*}{ HSV-1 } & $\mathrm{PI}$ & & 0.027 & 0.266 & 0.196 & 0.207 & $0.318^{*}$ & $0.325^{*}$ \\
\hline & Healthy & $340^{* *}$ & 0.088 & -0.047 & 0.185 & $0.233^{*}$ & $0.198^{*}$ & 0.144 \\
\hline
\end{tabular}

${ }^{*} p<0.05 ;{ }^{* *} p<0.01$ 


\section{DISCUSSION}

Results of this study showed that qualitative profile of HSV-1 referring to the presence/absence of the virus between PI and healthy controls was not different due to similar frequency of the virus between cases and controls. Moreover, the quantitative analysis of HSV-1 and periopathogens showed that HSV-1-positive patients exhibited significantly higher levels of Pi and $\mathrm{Cr}$. In addition, increased HSV levels were positively correlated with Tf, Pm, Fn, and Cr in PI as well as with $\mathrm{Aa}, \mathrm{Pi}$, and Pm in healthy controls, thus indicating the key impact of HSV on the quantitative profiling of the microflora. Finally, the HSV load of viral copies, as well as periopathogens levels, was significantly higher in peri-implant pockets compared with the internal implant portions.

The association of herpes virus with PI was previously investigated, and higher prevalence of the virus was reported for patients with PI when compared with participants with healthy peri-implant tissues.,11,16

This study established nonsignificant difference in prevalence of HSV-1 between PI and HI, disagreeing with the outcome reported by the study mentioned above. Such discrepancy can be explained by two possible facts: The sample size in the present study counted 40 patients with 77 implants affected by PI, i.e., roughly 3-fold greater than in the previous studies that counted 20 to 30 PI cases. In addition, the RT-PCR method used in this study is a more sensitive method than nested PCR used in the studies above mentioned, so that it may be possible that some cases in these studies were false negative. Generally speaking, since $95 \%$ of the population is infected with herpes virus, it would be difficult to establish specific relation only between its presence and PI. In the spirit of that, in the recently proposed keystone pathogens, hypothesis of periodontal disease was emphasized that the presence of periopathogens is similar between disease and health, while their quantitative interrelation determines the state. ${ }^{13}$ The findings from this study supported this hypothesis because similar qualitative profile of HSV-1 between PI and HI, but significantly different quantitative profile of HSV-1 and periopathogens between PI and HI have been found. Although in another study ${ }^{7}$ the levels of EBV had been correlated with the presence of periopathogens, the present is the first study that investigated the quantitative profile of interrelation between HSV-1 and periopathogens.

Moreover, the statistically significant increased PI and $\mathrm{Cr}$ in HSV-1-positive patients together with the correlation between HSV-1 and Tf, Pm, Fn, and Cr in PI further supports the proposed mechanism that HSV-1 and periopathogens create pathological synergism.

Furthermore, the obtained results may be also suggested that HSV provides favorable conditions for Tf, Pm,
Fn, and Cr overgrowth or that these microorganisms or some of them might stimulate reactivation of the virus.

These microorganisms have been correlated with PI, ${ }^{17}$ while the Fn was specifically associated with deepest peri-implant pockets ${ }^{18}$ and Fn together with Pm was associated with the pain in PI. ${ }^{19}$

Finally, Pm was reported as specifically associated with PI in a study that compared microflora between PI and periodontitis using advanced sequencing methods. ${ }^{20}$

The above-reported studies indicate that HSV-1 and periopathogens create bidirectional interaction in periodontitis and PI. It is suggested that periopathogens might lead to reactivation of HSV-1 from its latency, while HSV-1 further provides the favorable conditions for periopathogens overgrowth and manipulates host response enhancing the local inflammation. The HSV-1 provides synergistic and additive effects to periopathogens since they share similarity in the pathological pattern. Briefly, herpes viruses is recognized by Toll-like 2 receptors similarly as periopathogens that further induces release of cytokines, such as interleukin $1 \mathrm{~b}$ and tumor necrosis factor $\alpha$ via nuclear factor-kappa B transcription factor ${ }^{21}$ that have been previously reported to be associated with PI. ${ }^{22,23}$ Such an increase in proinflammatory cytokines might further increase receptor activator nuclear factor kappa-B ligand levels and, subsequently, enhance inflammatory osteoclastogenesis.

However, considering the extremely high prevalence in population of HSV-1 and the absence of correlation between HSV-1 and clinical parameters, it seems that the only presence of HSV-1 cannot be considered as a specific indicator of PI.

Increased HSV-1 counts were associated with increased levels of important periopathogens specifically implicated in PI pathology. It seems that HSV-1 plays the role as an enhancer of microbiological and immunological dysbiosis in peri-implant infection; hence, the quantitative profiling of this interrelation with periopathogens might provide accurate diagnostic information.

However, this hypothesis needs further comprehensive investigation. Further researches should be performed in a larger sample with the HSV-1 positivity as the primary grouping criterion. Moreover, the immunological and biochemical markers should be compared between HSV-1-positive and HSV-1-negative cases to investigate whether HSV-1 enhances local inflammation and tissue destruction.

\section{CONCLUSION}

As HSV-1 was found in similar levels of PI and HI patients after an average of 6 years of loaded implants, HSV-1 prevalence cannot be used to identify implants with or without the presence of PI. However, the 
correlation was identified between HSV-1 levels and certain periodontopathogens.

\section{CLINICAL SIGNIFICANCE}

The HSV-1 is identified in PI site and healthy site and the periopathogen levels only alter the bone support around the implant causing PI rather than herpes viruses. Thus, HSV-1 may represent a rather unspecific indicator for the host response to the bacterial challenge observed in PI.

\section{REFERENCES}

1. Rosen P, Clem D, Cochran D, Froum S, McAllister B, Renvert $S$, Wang HL. Peri-implant mucositis and peri-implantitis: a current understanding of their diagnoses and clinical implications. J Periodontol 2013 Apr;84(4):436-443.

2. Mombelli A, Décaillet F. The characteristics of biofilms in periimplant disease. J Clin Periodontol 2011 Mar;38 (Suppl 11): 203-213.

3. Zhuang LF, Watt RM, Mattheos N, Si MS, Lai HC, Lang NP. Periodontal and peri-implant microbiota in patients with healthy and inflamed periodontal and peri-implant tissues. Clin Oral Implants Res 2016 Jan;27(1):13-21.

4. Dabdoub SM, Tsigarida AA, Kumar PS. Patient-specific analysis of periodontal and peri-implant microbiomes. J Dent Res 2013 Dec;92(12 Suppl):168S-175S.

5. Berglundh T, Zitzmann NU, Donati M. Are peri-implantitis lesions different from periodontitis lesions? J Clin Periodontol 2011 Mar;38 Suppl 11:188-202.

6. Rotola A, Cassai E, Farina R, Caselli E, Gentili V, Lazzarotto T, Trombelli L. Human herpesvirus 7, Epstein-Barr virus and human cytomegalovirus in periodontal tissues of periodontally diseased and healthy subjects. J Clin Periodontol 2008 Oct;35(10):831-837.

7. Verdugo F, Castillo A, Castillo F, Uribarri A. Epstein-Barr virus associated peri-implantitis: a split-mouth study. Clin Oral Investig 2015 Mar;19(2):535-543.

8. Michalowicz BS, Ronderos M, Camara-Silva R, Contreras A, Slots J. Human herpesviruses and Porphyromonas gingivalis are associated with juvenile periodontitis. J Periodontol 2000 Jun;71(6):981-988.

9. Cohen JI. Epstein-Barr virus infection. N Engl J Med 2000 Aug;343(7):481-492.

10. Slots J. Herpesviral-bacterial interactions in periodontal diseases. Periodontol 20002010 Feb;52(1):117-140.
11. Jankovic S, Aleksic Z, Dimitrijevic B, Lekovic V, Camargo P, Kenney B. Prevalence of human cytomegalovirus and EpsteinBarr virus in subgingival plaque at peri-implantitis, mucositis and healthy sites. A pilot study. Int J Oral Maxillofac Surg 2011 Mar;40(3):271-276.

12. Canullo L, Pesce P, Pailler N, Simonetti M, Rakic M, Jovanovic S. The prevalence and quantitative analysis of the EpsteinBarr virus in healthy implants and implants affected by periimplantitis: a preliminary report. J Oral Sci Rehabil 2015 Oct;1(1):34-40.

13. Hajishengallis G. The inflammophilic character of the periodontitis-associated microbiota. Mol Oral Microbiol 2014 Dec;29(6):248-257.

14. Lang NP, Joss A, Orsanic T, Gusberti FA, Siegrist BE. Bleeding on probing. A predictor for the progression of periodontal disease? J Clin Periodontol 1986 Jul;13(6):590-596.

15. Ainamo J, Bay I. Problems and proposals for recording gingivitis and plaque. Int Dent J 1975 Dec;25(4):229-235.

16. Jankovic S, Aleksic Z, Dimitrijevic B, Lekovic V, Milinkovic I, Kenney B. Correlation between different genotypes of human cytomegalovirus and Epstein-Barr virus and peri-implant tissue status. Aust Dent J 2011 Dec;56(4):382-388.

17. Rakic M, Grusovin MG, Canullo L. The microbiologic profile associated with peri-implantitis in humans: a systematic review. Int J Oral Maxillofac Implants 2016 Mar-Apr;31(2): 359-368.

18. Renvert S, Roos-Jansåker AM, Lindahl C, Renvert H, Rutger Persson G. Infection at titanium implants with or without a clinical diagnosis of inflammation. Clin Oral Implants Res 2007 Aug;18(4):509-516.

19. Tabanella G, Nowzari H, Slots J. Clinical and microbiological determinants of ailing dental implants. Clin Implant Dent Relat Res 2009 Mar;11(1):24-36.

20. Koyanagi T, Sakamoto M, Takeuchi Y, Maruyama N, Ohkuma M, Izumi Y. Comprehensive microbiological findings in peri-implantitis and periodontitis. J Clin Periodontol 2013 Mar;40(3):218-226.

21. Khan G. Epstein-Barr virus, cytokines, and inflammation: a cocktail for the pathogenesis of Hodgkin's lymphoma? Exp Hematol 2006 Apr;34(4):399-406.

22. Duarte PM, de Mendonça AC, Máximo MB, Santos VR, Bastos MF, Nociti FH. Effect of anti-infective mechanical therapy on clinical parameters and cytokine levels in human peri-implant diseases. J Periodontol 2009 Feb;80(2):234-243.

23. Renvert S, Widén C, Persson GR. Cytokine expression in periimplant crevicular fluid in relation to bacterial presence. JClin Periodontol 2015 Jul;42(7):697-702. 